\title{
Premorbid IQ estimates obtained by combining the NART and demographic variables: construct validity
}

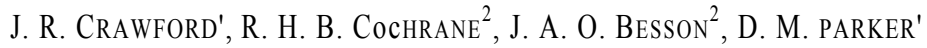 \\ and L. E. STEWART ${ }^{2}$ \\ Departments of ${ }^{\wedge}$ Psychology and $\wedge^{\wedge}$ Mental Health, University of Aberdeen, Aberdeen AB9 2UB, Scotland
}

(Received 12 April 1989)

\begin{abstract}
Summary - Crawford, Stewart, Parker, Besson \& Cochrane (Personality and Individual Differences, 10, 793-796, 1989a) have recently built regression equations to estimate premorbid IQ from the National Adult Reading Test (Nelson, 1982) and demographic variables. The construct validity of this IQ estimate was examined in a sample of 151 non-clinical subjects. Factor analysis was performed on WAIS subtests and the NART/demographic estimate (NDE). The loadings of these variables on the first unrelated principal component (which represents $g$ or general intelligence) was examined. The $g$ loading of the NDE was 0.90 , which was higher than any of the WAIS subtests. This result suggests that the NDE has very high construct validity and is superior to the NART alone as a measure of premorbid $g$.
\end{abstract}

\section{INTRODUCTION}

Attempting to detect and quantify intellectual impairment in the individual case is problematic. As there are substantial individual differences in intellectual ability in the general population, simply comparing a client's IQ test performance with the relevant norms will be of little value. It is therefore necessary to compare current performance against an individualised comparison standard. This comparison standard is easily obtained if test results are available from a period preceding the point at which behaviour change or neurological disorder raised the possibility of impairment. However, such information is rarely available. It is therefore necessary to estimate an individual's premorbid or expected level of ability.

One approach to obtaining this estimate is to examine performance on current ability measures relatively resistant to the effects of neurological or psychiatric disorder. The most commonly used test for this purpose is the National Adult Reading Test (NART; Nelson, 1982). This test consists of 50 words which Ss have to read and pronounce. The NART would appear to meet the three criteria necessary for a measure of premorbid IQ, i.e. it has high reliability, is capable of predicting a substantial proportion of IQ variance (in the normal population) and is largely resistant to neurological or psychiatric disorder (Crawford, 1989). An alternative approach is to estimate premorbid IQ from demographic variables. Wilson, Rosenbaum, Brown, Rourke, Whitman \& Grisell (1978), using the WAIS standardisation sample, built a regression equation based on five demographic variables (age, sex, race, education and occupation) which predicted 53\% of WAIS IQ variance.

In a recent study, Crawford et al. (1989a) attempted to improve the accuracy of premorbid IQ estimation by combining the above approaches. They built a multiple regression based on the NART and demographic variables and reported that this equation predicted significantly more IQ variance than either the NART or demographic variables alone.

The purpose of the present study was to determine the construct validity of the IQ estimate provided by the NART/demographic equation (NDE) by examining its loading on $g$.

\section{METHOD}

The sample consisted of the 151 Ss used to build the NART/demographic equations (all Ss were free of neurological, psychiatric or sensory disorder). Ss had been administered the NART and a full WAIS. A full description of this sample can be found in Crawford et al. (1989a). For each S, NART error score, sex, social class and age were entered into the NDE to provide the IQ estimate. Principal components analysis was then performed on this variable and the age-graded scaled scores of the 11 WAIS subtests.

\section{RESULTS AND DISCUSSION}

The first unrotated principal component $(\mathrm{g})$ accounted for $51.5 \%$ of the total test variance (Eigen value $=6.18)$. The $g$ loadings of the NDE estimate and the 11 WAIS subtests are presented in Table 1. It can be seen that the NDE IQ estimate loaded very highly on $g(0.90)$. Indeed, this loading was higher than any of the WAIS subtests.

This result compares favourably with the results obtained in a previous factor analytic study of the NART and WAIS (Crawford, Stewart, Cochrane, Parker \& Besson, 1989c). Although this study established that the NART is a very respectable measure of $g$, the loading obtained $(0.85)$ was exceeded by the Vocabulary and Information subtests and is lower than that obtained here by combining the NART with mediating demographic variables.

On the basis of the present results and the evidence that NART performance is largely resistant to the effects of neurological and psychiatric disorder, it can be concluded that the NART/demographic equation has very high construct validity as a measure of premorbid $g$. It therfore should be viewed as the method of choice in estimating premorbid intelligence. However, regression equations based solely on demographic variables (e.g. Wilson et al., 1978; Barona, Reynolds \& Chastain. 1984; Crawford, Stewart, Cochrane, Foulds, Besson \& Parker, 1989b) still have an obvious role in estimating premorbid IQ in patients for whom the NART, and therefore the NDE, would be inappropriate (e.g. patients with a marked dysarthria or dyslexia). 


\begin{tabular}{lc}
$\begin{array}{l}\text { Table 1. Loadings of WAIS subtests and the } \\
\text { NART/demographic estimate on the first unrotated princi- } \\
\text { pal component }(g)\end{array}$ \\
\hline \multicolumn{2}{c}{$g$ loadings } \\
\hline NART/demographic estimate & 0.90 \\
Information & 0.89 \\
Comprehension & 0.81 \\
Arithmetic & 0.71 \\
Similarities & 0.76 \\
Digit Span & 0.44 \\
Vocabulary & 0.88 \\
Digit Symbol & 0.54 \\
Picture Completion & 0.64 \\
Block Design & 0.72 \\
Picture Arrangement & 0.54 \\
Object Assembly & 0.59 \\
\hline
\end{tabular}

REFERENCES

Barona, A., Reynolds, C. R. \& Chastain, R. (1984). A demographically based index of premorbid intelligence for the WAIS-R. Journal of Consulting Clinical Psychology, 52, 885-877. Crawford, J. R. (1989). The estimation of premorbid intelligence: A review. In Developments in Clinical and Experimental

Neuropsychology (Edited by Crawford, J. R. \& Parker, D. M.). New York: Plenum Press. Crawford, J. R., Stewart, L. E., Parker, D. M., Besson, J. A. O. \& Cochrane, R. H. B. (1989a). Estimation of premorbid intelligence: Combining psychometric and demographic approaches improves predictive accuracy. Personality and Individual Differences, 10, 793-796. Crawford, J. R., Stewart, L. E., Cochrane, R. H. B., Foulds, J. A., Besson, J. A. O. \& Parker, D. M. (1989b). Estimating

premorbid IQ from demographic variables: Regression equations derived from a UK sample. British Journal of Clinical Psychology, 28, 275-278. Crawford, J. R., Stewart, L. E., Cochrane, R. H. B., Parker, D. M. \& Besson, J. A. O.

(1989c). Construct validity of the

National Adult Reading Test: A factor analytic study. Personality and Individual Differences 10, 585-587. Nelson,

H. E. (1982). National Adult Reading Test (NART): Test Manual. NFER-Nelson, Windsor. Wilson, R. S., Rosenbaum,

G., Brown, G., Rourke, D., Whitman, D. \& Grisell, J. (1978) An index of premorbid intelligence.

Journal Consulting Clinical Psychology, 46, 1554-1555. 